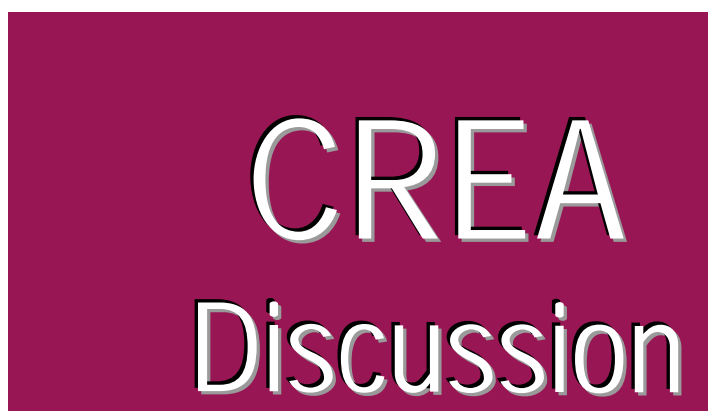
Paper

\title{
2014-27
}

Center for Research in Economics and Management

University of Luxembourg

\section{Essential Inputs and Unbounded Output: an Alternative Characterization of the Neoclassical Production Function}

available online : http://wwwfr.uni.Iu/recherche/fdef/crea/publications2/discussion_papers

Andreas Irmen, CREA, Université de Luxembourg and CESifo, Munich

Alfred Maußner, University of Augsburg

November, 2014

For editorial correspondence, please contact: crea@uni.lu

University of Luxembourg

Faculty of Law, Economics and Finance

162A, avenue de la Faïencerie

L-1511 Luxembourg 


\title{
ESSENTIAL INPUTS AND UNBOUNDED OUTPUT: AN ALTERNATIVE CHARACTERIZATION \\ OF THE \\ NEOCLASSICAL PRODUCTION FUNCTION
}

\author{
Andreas Irmen* \\ University of Luxembourg and CESifo, Munich \\ Alfred Maußner ${ }^{\dagger}$ \\ University of Augsburg
}

\begin{abstract}
The Inada (1963) conditions constitute a defining property of the neoclassical production function with capital and labor as arguments. Are these conditions justifiable on economic grounds? Yes, they are: we show that a production function with positive, yet diminishing marginal products and constant returns to scale satisfies the Inada conditions if i) both inputs are essential and ii) an unbounded quantity of either input leads to unbounded output. This allows for an alternative characterization of the neoclassical production function that altogether dispenses with the Inada conditions. Moreover, we establish that the marginal product of capital vanishes as capital goes to infinity if labor is an essential input. Given the intuitive appeal of the latter feature, we conclude that the neoclassical growth model is a theory of eventual stagnation.
\end{abstract}

Keywords: Neoclassical Growth Model, Capital Accumulation, Stagnation, Inada Conditions.

JEL-Classification: E10, O10, O40.

This Version: November 28, 2014.

\footnotetext{
${ }^{*}$ Corresponding Author. Address: University of Luxembourg, CREA, Faculty of Law, Economics and Finance, 162a, avenue de la Faïencerie L-1511 Luxembourg, Luxembourg, airmen@uni.lu.

${ }^{\dagger}$ University of Augsburg, Chair of Empirical Macroeconomics, Universitätsstrasse 16, D-86316 Augsburg, Germany, alfred.maussner@wiwi.uni-augsburg.de.

We are grateful to Hendrik Hakenes, Amer Tabaković, and Gautam Tripathi for useful comments and suggestions.
} 


\section{Introduction}

The neoclassical production function is a corner stone of neoclassical growth theory and of modern dynamic macroeconomics in general. According to Barro and Sala-í-Martin (2004), Chapter 1, a production function taking capital and labor as arguments is called neoclassical if it exhibits three defining properties: positive and diminishing marginal products of both inputs, constant returns to scale, and the Inada conditions. ${ }^{1}$ While the first two properties have convincing intuitive appeal, the main justification given for the Inada conditions is simply analytical convenience. ${ }^{2}$

The main purpose of this note is to show that an intuitive justification for the Inada conditions can be given. More precisely, our main result states that the Inada conditions hold if both capital and labor are essential and if an unbounded quantity of either input leads to unbounded output. Using this finding we come up with an alternative, yet equivalent, definition of the neoclassical production function that altogether dispenses with the Inada conditions.

To accomplish this our analysis proceeds in three steps. First, we show in Section 2 that the Inada condition at infinity for one input is implied by the essentiality of the other input. We emphasize in passing that this result has an important implication for the long-run prediction of the neoclassical growth model: if labor is an essential input in the aggregate production function then the marginal product of capital goes to zero as capital goes to infinity and the neoclassical growth model is a theory of eventual stagnation. ${ }^{3}$

Section 3 establishes sufficient conditions for the Inada conditions at zero. They include the condition of essential inputs in conjunction with the requirement that output becomes unbounded as either input goes to infinity. Section 4 has our main result. We prove for a production function with positive and diminishing marginal products of both inputs and constant returns to scale that the (four) Inada conditions are equivalent to a set of four conditions: capital and labor are essential and an unbounded input of either of these

\footnotetext{
${ }^{1}$ Some authors such as Acemoglu (2009), Chapter 2, or Romer (2012), Chapter 1, include the essentiality of capital to this set of properties. However, the three defining properties mentioned above can be shown to imply this feature (see, Barro and Sala-1-Martin (2004), Chapter 1).

${ }^{2}$ The Inada conditions prescribe that the limit of the marginal products of capital and labor is infinity (zero) as the respective input approaches zero (infinity). In many circumstances, these conditions are sufficient for the existence of interior equilibria. Inada himself does not provide an economic justification for his conditions either but simply refers to them as "derivative conditions" (Inada (1963), p. 121).

${ }^{3}$ The term "neoclassical growth model" refers to the class of mathematical models that studies the process of capital accumulation and its implications for economic growth. The contributions that develop the analytical core of these models include Ramsey (1928), Solow (1956), Swan (1956), Cass (1965), and Koopmans (1965).
} 
inputs leads to unbounded output. Finally, we use this finding for an alternative characterization of the neoclassical production function. Proofs are given in the main text except those of Proposition 1 and 2 which are relegated to Section 5, the Appendix.

\section{Essential Inputs and the Inada Conditions at Infinity}

Throughout this note we study aggregate production functions $F(K, L)$ where $K>0$ and $L>0$ denote the inputs of capital and labor. These functions satisfy the following properties.

Definition 1 (Aggregate Production Function)

The aggregate production function, $F: \mathbb{R}_{++}^{2} \rightarrow \mathbb{R}_{++}$,

1. is twice differentiable with positive, yet diminishing marginal products, i.e.,

$$
F_{K}(K, L)>0>F_{K K}(K, L) \text { and } F_{L}(K, L)>0>F_{L L}(K, L) \text {, }
$$

2. exhibits constant returns to scale in $K$ and $L$.

For functions that comply with Definition 1 we now establish that essential inputs are sufficient for the Inada conditions at infinity. To introduce the notion of an essential input, let $F(K, 0) \equiv \lim _{L \rightarrow 0} F(K, L)$ and $F(0, L) \equiv \lim _{K \rightarrow 0} F(K, L)$. Then, labor is an essential input, or essential for short, if $F(K, 0)=0$, and capital is essential if $F(0, L)=0$.

Proposition 1 (Essential Inputs and Inada Conditions at Infinity)

1. If labor is essential then $\lim _{K \rightarrow \infty} F_{K}(K, L)=0$.

2. If capital is essential then $\lim _{L \rightarrow \infty} F_{L}(K, L)=0$.

Hence, essentiality of one input implies the Inada condition at infinity of the other input. To grasp the intuition for this finding consider Claim 1. Since labor is essential, the average product of capital must vanish as labor converges to zero, i. e., for $L \rightarrow 0$ we have $F(K, L) / K \rightarrow 0$. Under constant returns to scale the latter implies that $f(k) / k \rightarrow 0$ as $k \rightarrow \infty$, where $f(k) \equiv F(k, 1)$ and $k \equiv K / L$. By assumption, the marginal product of labor is strictly positive so that $F_{L}(K, L)=f(k)-k f^{\prime}(k)>0$. This requires the average product of capital to be strictly greater than its marginal product, i. e., $f(k) / k>f^{\prime}(k)$. 
As $f(k) / k$ tends to zero as $L \rightarrow 0, f^{\prime}(k)=F_{K}(K, L)$ must also tend to zero. Hence, the Inada condition at infinity for capital is satisfied if labor is essential. ${ }^{4}$ Mutatis mutandis, an analogous intuition may be developed for Claim 2.

Proposition 1 gives rise to an important corollary that links the long-run predictions of the neoclassical growth model to the essentiality of labor.

\section{Corollary 1 (Essential Labor and Long-Run Stagnation)}

For the neoclassical growth model with an aggregate production function of Definition 1 the following holds:

If labor is essential then the neoclassical growth model is a theory of eventual stagnation.

The intuition for Corollary 1 is straightforward. To exhibit long-run growth the neoclassical growth model needs to eliminate or at least to weaken the tendency of diminishing returns to capital (see, e. g., Solow (1956), p.77, and de La Grandville (1989), p.467-469). However, from Claim 1 of Proposition 1 we know that this cannot happen if labor is an essential input since then $\lim _{K \rightarrow \infty} F_{K}(K, L)=0$.

\section{Unbounded Output, Essential Inputs, and the Inada Condi- tions at Zero}

In addition to essential inputs the Inada conditions at zero require that output approaches infinity as either input goes to infinity. We capture this property using the following notation: $F(K, \infty) \equiv \lim _{L \rightarrow \infty} F(K, L)=\infty$ and $F(\infty, L) \equiv \lim _{K \rightarrow \infty} F(K, L)=\infty$.

Proposition 2 (Unbounded Output, Essential Inputs, and Inada Conditions at Zero)

1. If $F(K, \infty)=\infty$ and capital is essential then $\lim _{K \rightarrow 0} F_{K}(K, L)=\infty$.

\footnotetext{
${ }^{4}$ To illustrate the claims of Proposition 1 consider the following two production functions that are both consistent with Definition 1 (see, de la Croix and Michel (2002), p.122, and Litina and Palivos (2008), p. 498, respectively). First, let $F(K, L)=b L+a L K /(L+K), a, b>0$. Here, labor is essential and capital is not. Accordingly, we have $\lim _{K \rightarrow \infty} F_{K}(K, L)=\lim _{K \rightarrow \infty} a(L /(L+K))^{2}=0$ whereas $\lim _{L \rightarrow \infty} F_{L}(K, L)=\lim _{L \rightarrow \infty} b+a(K /(K+L))^{2}=b>0$. Second, let $F(K, L)=A K+\alpha L-\beta L \exp (-\beta K / L)$, $A>0, \alpha \geq \beta>0$. Here, capital is essential if $\alpha=\beta$ in which case $\lim _{L \rightarrow \infty} F_{L}(K, L)=$ $\lim _{L \rightarrow \infty} \alpha(1-(1+\alpha K / L) \exp (-\alpha K / L))=0$. Interestingly, for $\alpha>\beta$ capital is no longer essential and the conclusion of Claim 2 is no longer valid as $\lim _{L \rightarrow \infty} F_{L}(K, L)=\alpha-\beta>0$.
} 
2. If $F(\infty, L)=\infty$ and labor is essential then $\lim _{L \rightarrow 0} F_{L}(K, L)=\infty$.

Hence, the Inada condition at zero of one input obtains if this input is essential and output becomes unbounded as the other input tends to infinity. To develop the intuition for this finding consider Claim 1. If output becomes unbounded as labor goes to infinity then the average product of capital becomes unbounded, too, i.e., for $L \rightarrow \infty$ we have $F(K, L) / K \rightarrow \infty$. Under constant returns to scale the latter implies that $f(k) / k \rightarrow \infty$ as $k \rightarrow 0$. Since capital is essential, it holds that $f(k) \rightarrow 0$ as $k \rightarrow 0$. Therefore, the limit of the average product of capital as $k \rightarrow 0$ is equal to the limit of the marginal product of capital and both are equal to infinity. Since $f^{\prime}(k)=F_{K}(K, L)$ it follows that the Inada condition at zero for capital is satisfied. ${ }^{5}$ Again, mutatis mutandis, the intuition for Claim 2 is analogous.

\section{An Alternative Characterization of the Neoclassical Produc- tion Function}

What are the implications of Proposition 1 and 2 for the notion of a neoclassical production

function? The formal definition of this concept is the following (see, Barro and Sala-1Martin (2004), p. 27-28).

Definition 2 (Neoclassical Production Function)

An aggregate production function of Definition 1 is called a neoclassical production function if it also satisfies the Inada conditions

$$
\begin{aligned}
& \lim _{K \rightarrow 0} F_{K}(K, L)=\lim _{L \rightarrow 0} F_{L}(K, L)=\infty, \\
& \lim _{K \rightarrow \infty} F_{K}(K, L)=\lim _{L \rightarrow \infty} F_{L}(K, L)=0 .
\end{aligned}
$$

To establish an alternative and equivalent definition to Definition 2 we first use Proposition 1 and 2 to state and prove the following result.

\footnotetext{
${ }^{5}$ Observe that for capital's (labor's) Inada condition at zero it is not sufficient that capital (labor) is essential. To see this consider Claim 1 of Proposition 2 and $F(K, L)=L(1-\exp (-\beta K / L)), \beta>0$, which satisfies Definition 1. Here, capital is essential, yet $\lim _{K \rightarrow 0} F_{K}(K, L)=\beta<\infty$. However, this function does not satisfy the "unbounded output" requirement since $F(K, \infty)=\lim _{L \rightarrow \infty} F(K, L)=\beta K<\infty$.
} 
Proposition 3 (Essential Inputs, Unbounded Output, and Inada Conditions)

An aggregate production function of Definition 1 satisfies the set of Inada conditions (4.1) if and only if it satisfies

$$
\begin{array}{r}
F(0, L)=F(K, 0)=0, \\
F(K, \infty)=F(\infty, L)=\infty .
\end{array}
$$

In other words, an aggregate production function of Definition 1 will satisfy the Inada conditions if and only if both inputs are essential and output becomes unbounded if one input becomes unbounded.

The proof and the intuition behind Proposition 3 are straightforward. In fact the "if part" follows directly from Proposition 1 and 2. From Proposition 1 we know that $F(0, L)=$ $F(K, 0)=0$ implies $\lim _{K \rightarrow \infty} F_{K}(K, L)=\lim _{L \rightarrow \infty} F_{L}(K, L)=0$. Proposition 2 states that the same essentiality conditions in conjunction with unbounded output, i. e., $F(K, \infty)=$ $F(\infty, L)=\infty$, imply $\lim _{K \rightarrow 0} F_{K}(K, L)=\lim _{L \rightarrow 0} F_{L}(K, L)=\infty$. Moreover, the converse implications are established in Barro and Sala-í-Martin (2004), p.77-78. Hence, Proposition 3 holds.

Proposition 3 leads to the main result of this note. We state our alternative definition of the neoclassical production function as Corollary 2.

Corollary 2 (Neoclassical Production Function without Inada Conditions)

Consider an aggregate production function of Definition 1. This function is a neoclassical production function in the sense of Definition 2 if it satisfies the conditions (4.2).

Hence, the alternative definition of the neoclassical production function that we propose replaces the four Inada conditions by four new conditions: each input is essential and output becomes unbounded if either input tends to infinity. 


\section{Appendix: Proofs}

\subsection{Proof of Proposition 1}

Claim 1 Let $k \equiv K / L$ and $F(k, 1) \equiv f(k)$. Since $(K, L) \in \mathbb{R}_{++}^{2}$, we have $k \in \mathbb{R}_{++}$. Then, it holds that

$$
\lim _{L \rightarrow 0} F(K, L)=\lim _{L \rightarrow 0} L f(k)=K \lim _{L \rightarrow 0} \frac{f(k)}{k} .
$$

From the definition of $k$ it follows that $\lim _{L \rightarrow 0} k=\lim _{k \rightarrow \infty} k=\infty$. Hence, if labor is essential then

$$
0=\lim _{L \rightarrow 0} \frac{f(k)}{k}=\lim _{k \rightarrow \infty} \frac{f(k)}{k} .
$$

Since the marginal product of labor is strictly positive we have $F_{L}(K, L)=f(k)-k f^{\prime}(k)>$ 0 . As a consequence, $f(k) / k>f^{\prime}(k)$, and with (5.1) we obtain

$$
0=\lim _{k \rightarrow \infty} \frac{f(k)}{k} \geq \lim _{k \rightarrow \infty} f^{\prime}(k)=\lim _{K \rightarrow \infty} F_{K}(K, L),
$$

where the last equality follows since $f^{\prime}(k)=F_{K}(K, L)$ for all $K$ and $L$. Hence, if labor is essential then the Inada condition $\lim _{K \rightarrow \infty} F_{K}(K, L)=0$ holds.

Claim 2 Let $l \equiv L / K$ and $F(1, l) \equiv v(l)$. Since $(K, L) \in \mathbb{R}_{++}^{2}$, we have $l \in \mathbb{R}_{++}$. Then, it holds that

$$
\lim _{K \rightarrow 0} F(K, L)=\lim _{K \rightarrow 0} K v(l)=L \lim _{K \rightarrow 0} \frac{v(l)}{l} .
$$

From the definition of $l$ it follows that $\lim _{K \rightarrow 0} l=\lim _{l \rightarrow \infty} l=\infty$. Hence, if capital is essential then

$$
0=\lim _{K \rightarrow 0} \frac{v(l)}{l}=\lim _{l \rightarrow \infty} \frac{v(l)}{l} .
$$

Since the marginal product of capital is strictly positive we have $F_{K}(K, L)=v(l)-$ $l v^{\prime}(l)>0$. As a consequence, $v(l) / l>v^{\prime}(l)$, and with (5.2) we obtain

$$
0=\lim _{l \rightarrow \infty} \frac{v(l)}{l} \geq \lim _{l \rightarrow \infty} v^{\prime}(l)=\lim _{L \rightarrow \infty} F_{L}(K, L),
$$

where the last equality follows since $v^{\prime}(l)=F_{L}(K, L)$ for all $K$ and $L$. Hence, if capital is essential then the Inada condition $\lim _{L \rightarrow \infty} F_{L}(K, L)=0$ holds. 


\subsection{Proof of Proposition 2}

Claim 1 For any $K \in \mathbb{R}_{++}$it holds that

$$
\lim _{L \rightarrow \infty} F(K, L)=\lim _{L \rightarrow \infty} L f(k)=K \lim _{L \rightarrow \infty} \frac{f(k)}{k} .
$$

From the definition of $k$ it follows that $\lim _{L \rightarrow \infty} k=\lim _{k \rightarrow 0} k=0$. Hence, if output approaches infinity as labor goes to infinity then

$$
\infty=\lim _{L \rightarrow \infty} \frac{f(k)}{k}=\lim _{k \rightarrow 0} \frac{f(k)}{k} .
$$

Since capital is essential we have for any $L \in \mathbb{R}_{++}$that

$$
0=\lim _{K \rightarrow 0} F(K, L)=L \lim _{K \rightarrow 0} f(k)=\lim _{k \rightarrow 0} f(k) .
$$

Therefore, using l'Hôpital's rule in (5.3) delivers

$$
\infty=\lim _{k \rightarrow 0} \frac{f(k)}{k}=\lim _{k \rightarrow 0} f^{\prime}(k)=\lim _{K \rightarrow 0} F_{K}(K, L),
$$

where the last equality follows since $f^{\prime}(k)=F_{K}(K, L)$ for all $K$ and $L$.

Hence, if output approaches infinity as labor goes to infinity and capital is essential then the Inada condition $\lim _{K \rightarrow 0} F_{K}(K, L)=\infty$ holds.

Claim 2 For any $L \in \mathbb{R}_{++}$it holds that

$$
\lim _{K \rightarrow \infty} F(K, L)=\lim _{K \rightarrow \infty} K v(l)=L \lim _{K \rightarrow \infty} \frac{v(l)}{l} .
$$

From the definition of $l$ it follows that $\lim _{K \rightarrow \infty} l=\lim _{l \rightarrow 0} l=0$. Hence, if output approaches infinity as capital goes to infinity then

$$
\infty=\lim _{K \rightarrow \infty} \frac{v(l)}{l}=\lim _{l \rightarrow 0} \frac{v(l)}{l} .
$$

Since labor is essential we have for any $K \in \mathbb{R}_{++}$that

$$
0=\lim _{L \rightarrow 0} F(K, L)=K \lim _{L \rightarrow 0} v(l)=\lim _{l \rightarrow 0} v(l) .
$$

Therefore, using l'Hôpital's rule in (5.4) delivers

$$
\infty=\lim _{l \rightarrow 0} \frac{v(l)}{l}=\lim _{l \rightarrow 0} v^{\prime}(l)=\lim _{L \rightarrow 0} F_{L}(K, L),
$$

where the last equality follows since $v^{\prime}(l)=F_{L}(K, L)$ for all $K$ and $L$.

Hence, if output approaches infinity as capital goes to infinity and labor is essential then the Inada condition $\lim _{L \rightarrow 0} F_{L}(K, L)=\infty$ holds. 


\section{References}

Acemoglu, D. (2009): Introduction to Modern Economic Growth. Princeton University Press, Princeton, New Jersey.

BARro, R. J., AND X. SAlA-Í-MARTIN (2004): Economic Growth. MIT Press, Cambridge, MA, 2nd edn.

CASS, D. (1965): "Optimum Growth in an Aggregative Model of Capital Accumulation," Review of Economic Studies, 32, 233-240.

DE LA CRoix, D., And P. Michel (2002): A Theory of Economic Growth - Dynamics and Policy in Overlapping Generations. Cambridge University Press, Cambridge, UK.

De La Grandville, O. (1989): "In Quest of the Slutsky Diamond," American Economic Review, 79(3), 468-481.

KoOpmans, T. (1965): "On the Concept of Optimal Economic Growth," in (Study Week on) The Economic Approach to Development Planning, ed. by P. A. S. S. Varia, pp. 225-287. North Holland, Amsterdam.

LitinA, A., AND T. PAlivos (2008): “Do Inada Conditions Imply that Production Function Must be Asymptotically Cobb-Douglas? A Comment," Economics Letters, 99, 498499.

RAmseY, F. P. (1928): “A Mathematical Theory of Savings," The Economic Journal, 38, 543-559.

Romer, D. (2012): Advanced Macroeconomics. McGraw Hill, New York, 4th edn.

SolOw, R. M. (1956): "A Contribution to the Theory of Economic Growth," Quarterly Journal of Economics, 70(1), 65-94.

SWAN, T. W. (1956): "Economic Growth and Capital Accumulation," Economic Record, 32, 334-361. 